Rapi d fr equency st ep- swi t chi ng i n submi I I met er wave gyr ot rons ( Gyr ot rons FU I I I and FU I V)

\begin{tabular}{|l|l|}
\hline 著者 & $\begin{array}{l}\text { I DEHARA T, SH M ZU Y, OGAWA I, TATSUKAWA T, } \\
\text { BRAND G. F. }\end{array}$ \\
\hline $\begin{array}{l}\text { j our nal or } \\
\text { publ i cat } \mathrm{i} \text { on } \mathrm{t} \text { i t l e }\end{array}$ & Physi cs of PI asmas \\
\hline vol une & 6 \\
\hline number & 6 \\
\hline page r ange & $2613-2617$ \\
\hline year & $1999-06$ \\
\hline URL & ht t p: //hdl . handl e. net /10098/1512 \\
\hline
\end{tabular}




\title{
Rapid frequency step-switching in submillimeter wave gyrotrons (Gyrotrons FU III and FU IV)
}

\author{
T. Idehara \\ Research Center for Development of Far-Infrared Region, Fukui University, Bunkyo 3-9-1, Fukui-shi 910 \\ 8507, Japan \\ Y. Shimizu \\ Department of Applied Physics, Faculty of Engineering, Fukui University, Bunkyo 3-9-1, \\ Fukui-shi 910-8507, Japan \\ 1. Ogawa \\ Cryogenic Laboratory, Faculty of Engineering, Fukui University, Bunkyo 3-9-1, Fukui-shi 910-8507, Japan \\ T. Tatsukawa \\ Department of Physics, Kagawa Medical University, Ikenobe 1750-1, Miki-cho, Kita-gun, \\ Kagawa-ken 761-0793, Japan \\ G. F. Brand \\ School of Physics, University of Sydney, NSW 2006, Australia
}

The rapid frequency step-switching between two nearby cyclotron fundamental modes achieved by switching beam energy is analyzed by a computer simulation and demonstrated experimentally. More dramatic frequency switching between a fundamental mode and a second harmonic mode is analyzed and demonstrated experimentally.

\section{INTRODUCTION}

Both frequency modulation and amplitude modulation of gyrotron output are important and useful for extending gyrotron applications to many fields. Amplitude modulation has been achieved in high power gyrotrons $\mathrm{s}^{1,2}$ and also in highfrequency gyrotrons. ${ }^{3-5}$ The principle is as follows. The anode voltage $V_{a}$ of a triode magnetron injection gun determines the pitch angle $\alpha$ of a beam electron in the gyrotron cavity, which in turn determines the efficiency of energy transfer from beam electrons to the electromagnetic wave Therefore, modulation of the anode voltage $V_{a}$ results in modulation of the output power from the gyrotron.

Our high-frequency gyrotrons covering the wide range from millimeter to submillimeter wavelengths (Gyrotrons FU III and FU IV) have demonstrated ${ }^{3,4}$ amplitude modulation of their outputs, where in some situations only a few percent modulation of $V_{a}$ causes almost $100 \%$ modulation of the output amplitude. Furthermore, a linear dependence between $V_{a}$ and the amplitude is realized over a wide range of gyrotron operating parameters. The modulation frequency can be increased up to several hundred $\mathrm{kHz}^{3,4}$ or several MHz. ${ }^{1}$ A beam with such a modulation may be applied to a phase sensitive measurement of plasma and other materials, communications in millimeter to submillimeter wave region, study of relaxation phenomena in plasma, and so on.

On the other hand, the frequency modulation of gyrotron output has also been achieved by one of our high-frequency gyrotrons (Gyrotron FU $\mathrm{IV}^{6,7}$ ). The principle is as follows. A gyrotron operates at a frequency $f$ near the electron cyclotron frequency $f_{c}$ or a harmonic $n f_{c}$. Here, $f_{c}=e B_{0} / 2 \pi m$, where $e$ is the electric charge, $m=g m_{0}$ the mass of the electron, $B_{0}$ the static magnetic field, $m_{0}$ the rest mass and $g=(1$ $\left.-v^{2} / c^{2}\right)^{-1 / 2}$ the relativistic factor. Therefore, the modulation of the cathode voltage $V_{b}$ of the electron gun causes a modulation of beam velocity, which in turn results in a change in the electron mass $m$ and then in modulation of $f_{c}$. This kind of frequency modulation of gyrotron output can only take place wilhin a frequency range restricted by the $Q$ of the resonant cavity. The amount of frequency modulation obtained is typically $20 \mathrm{MHz}$. The modulation efficiency $\Delta f / \Delta V_{b}$ is around $0.25 \mathrm{MHz} / \mathrm{V}$, which is smaller than the variation of electron cyclotron frequency $\Delta f_{c} / \Delta V_{b}$ by a factor of 2.3. This difference is likely to be due to the way the $Q$ of the resonant cavity restricts the operating frequency. ${ }^{6}$

In this paper, a different kind of frequency modulation, rapid frequency step-switching, is described. ${ }^{8,9}$ If there are two cavity modes whose operating frequencies are very close, it becomes easy to switch from one to the other by switching the cathode voltage $V_{b}$ to a new value. In this way, a rapid frequency step-switching of gyrotrons can be achieved. ${ }^{10}$ Here, we will describe both computer simulation and experimental resuits of such frequency step-switching between two electron cyclotron fundamental modes, and a similar but more dramatic step-switching between a fundamental and a second harmonic mode. The frequency modulation of gyrotron output may be useful for communications and scientific measurements.

\section{EXPERIMENTAL APPARATUS}

Figure 1 shows the experimental arrangement. The demountable gyrotron (Gyrotron FU $\mathrm{II}^{\mathrm{Il}}$ ) or the sealed-off gyrotron (Gyrotron FU IV ${ }^{12}$ ) have both been used. Both gyrotrons are frequency step tunable over a series of many resonances in the millimeter to submillimeter wavelength 


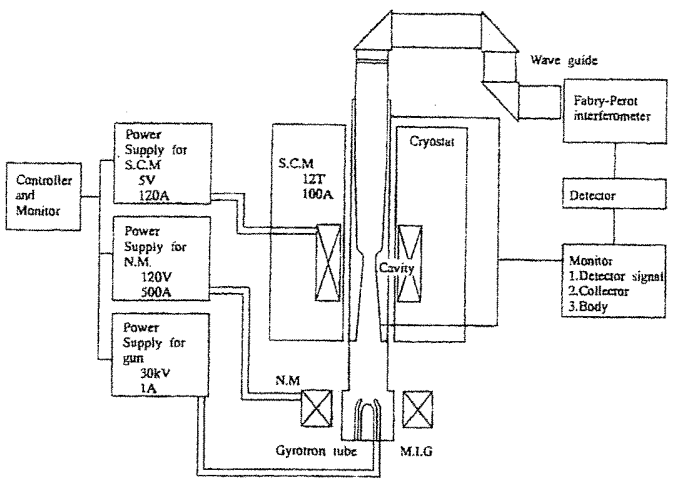

FIG. 1. The experimental arrangement including the gyrotron oscillator, power supplies, and measurement system.

zange. Gyrotrons FU III and FU IV have achieved frequency step tunabilities from 100 to $636 \mathrm{GHz}$ and from 160 to 847 $\mathrm{GHz}$, respectively.

A pulsed high-voltage power supply, which can deliver a maximum voltage of $30 \mathrm{kV}$ and a maximum current of $1 \mathrm{~A}$, is connected to the cathode of the gyrotron's triode magnetron injection gun. The voltage is divided by resistors and applied to the anode of gun. The pulse width can be varied up to $10 \mathrm{msec}$ and the voltage can be modulated up to $25 \%$. The modulation frequency can be varied up to $5 \mathrm{kHz}$. In the frequency step-switching experiment, square wave modulation was used.

We used a Fabry.Perot interferometer for frequency measurement. It can be used as a bandpass filter for separating one frequency from the other. This demonstrates clearly that the frequency step-switching occurs. Alternatively, when the cathode voltage is switched from $V_{b 1}$ to $V_{b 2}$, the frequency switching can be confirmed by sampling the output first while the voltage is $V_{b 1}$ and again while it is $V_{b 2}$, and measuring the frequencies in both cases by the FabryPerot interferometer.

\section{EXPERIMENTAL AND SIMULATION RESULTS FOR THE FREQUENCY STEP-SWITCHING BETWEEN TWO FUNDAMENTAL MODES}

For Gyrotron FU IV, Table I shows several cavity modes operating at the fundamental of the electron cyclotron frequency under the constant field intensity $B_{0}$ of $14.6 \mathrm{~T}$ and

TABLE 1. Several cavity modes $T E_{m n}$ which are able to operate at the magnetic field intensity $B_{0}$ of $14.6 \mathrm{~T}$, corresponding frequency $f$, and cathode voltage $V_{b}$

\begin{tabular}{ccc}
\hline \hline Cavity mode & Frequency $f(\mathrm{GHz})$ & Cathode voltage $V_{b}(\mathrm{kV})$ \\
\hline$T E_{43}$ & 372.511 & -49.629 \\
$T E_{111}$ & 376.755 & -43.315 \\
$T E_{72}$ & 379.863 & -38.779 \\
$T E_{24}$ & 386.848 & -28.852 \\
$T E_{04}$ & 391.348 & -22.644 \\
$T E_{112}$ & 407.643 & -1.312 \\
\hline
\end{tabular}

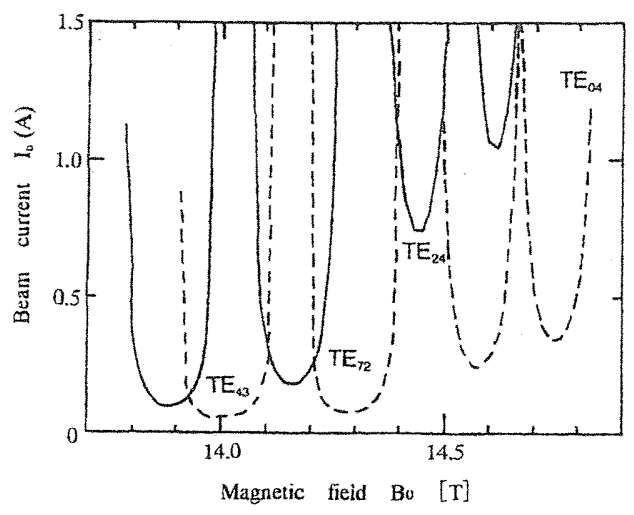

FIG. 2. Simulation results for beam current $I_{b}$ to sustain $100 \mathrm{~W}$ output power as functions of the magnetic field intensity $B_{0}$. Two cases are shown: $V_{b 1}=22.6$ (solid curves) and $V_{b 2}=28.8 \mathrm{kV}$ (broken curves).

their corresponding cathode voltages $V_{b}$. For our experiment, we have chosen the $T E_{241}$ and $T E_{041}$ modes. The cathode voltages can be provided by the available pulsed highvoltage power supply with maximum voltage of $30 \mathrm{kV}$.

For both cases of $V_{b 1}=28.8$ and $V_{b 2}=22.6 \mathrm{kV}$, com puter simulations give pitch angles $\alpha$ of 1.07 and 0.69 , respectively, and an injection radius of $0.81 \mathrm{~mm}$ for both cases.

These parameters are used for the subsequent calculation of energy transfer in the cavity from the beam electrons to high-frequency electromagnetic waves. Figure 2 shows the results in terms of the beam currents $I_{b}$, which are necessary to sustain $100 \mathrm{~W}$ output power for each cavity mode as funcbons of field intensity $B_{0}$. These results indicate that the $T E_{241}$ and $T E_{041}$ modes can indeed be switched if the cathode voltage $V_{b}$ is switched between 28.8 and $22.6 \mathrm{kV}$.

This is supported by a measurement of the output power by the pyroelectric detector current as a function of the cathode voltage $V_{b}$. The constant field intensity of $B_{0}$ is $14.6 \mathrm{~T}$. The result is shown in Fig. 3. When $V_{b}$ is increased, the cavity mode changes from $T E_{041}$ to $T E_{241}$ at around $V_{b}$

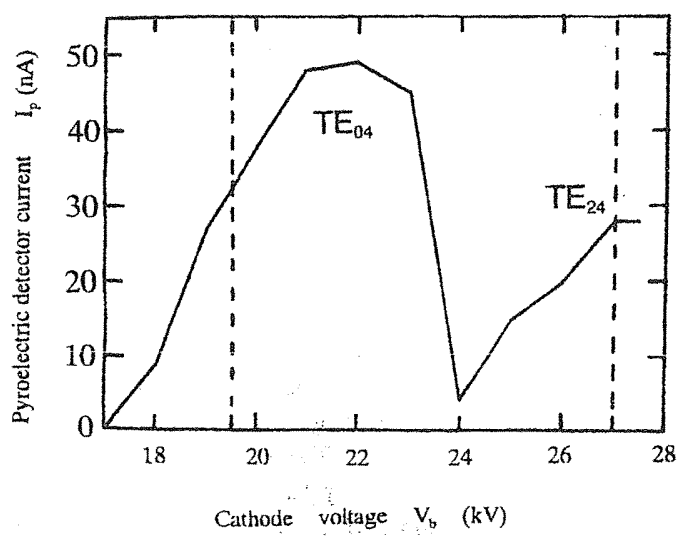

FIG. 3. Output power of $T E_{041}$ and $T E_{241}$ modes as measured by a pyro electric detector as a function of the cathode voltage $V_{b} . B_{0}=14.6 \mathrm{~T}$ 

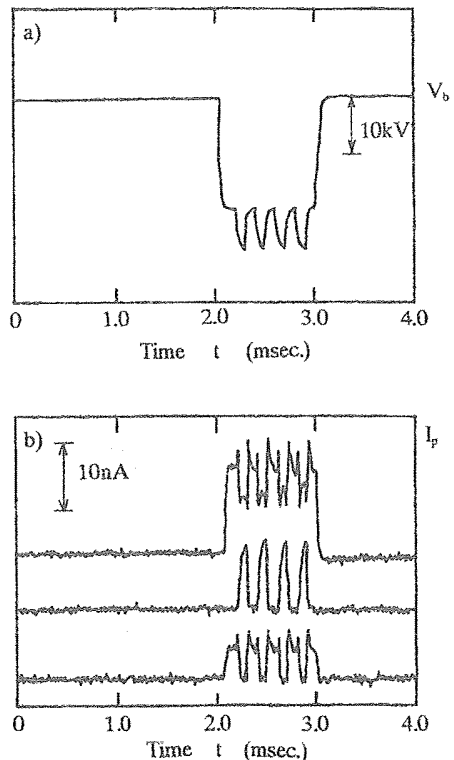

FIG. 4. (a) High-voltage pulse applied to the cathode of an electron gun, modulated by a $5 \mathrm{kHz}$ square wave. (b) Upper trace: Output power measured by a pyroelectric detector. Middle trace: The output power measured after passing through a Fabry-Perot interferoneter which is used as a bandpass filter for the $T E_{241}$ mode. Lower trace: The output power after the interferometer used as a bandpass filter for the $T E_{041}$ mode.

$=24 \mathrm{kV}$. If $V_{b}$ is switched from 19.5 to $27 \mathrm{kV}$ (indicated in Fig. 3 by dotted lines), the cavity mode should be switched from $T E_{041}$ to $T E_{241}$. This means that there should be a step-switching of the output frequency from 391 to $387 \mathrm{GHz}$.

Figure 4 shows a typical experimental result of the frequency step-switching where the cavity mode is switched. from $T E_{041}$ to $T E_{241}$. The cathode voltage is applied as a 1 msec pulse [shown in Fig. $4(\mathrm{a})$ ], and is switched between 19.5 and $27 \mathrm{kV}$. The switching frequency is around $5 \mathrm{kHz}$. The upper trace of Fig. 4(b) shows the output power measured by a pyroelectric detector. The output power at the phase of higher cathode voltage $\left(V_{b}=27 \mathrm{kV}\right)$ is separated from that at the lower cathode voltage $\left(V_{\bar{b}}=19.5 \mathrm{kV}\right)$ by using a Fabry-Perot interferometer as a bandpass filter. The middle and the lower traces show both the powers at the higher and the lower cathode voltages, respectively.

Figure 5 demonstrates the results of frequency measurements by sampling methods. The upper trace shows a Fabry. Perot interferometer pattern, when the gate pulse covers both phases of $V_{b}=19.5$ and $V_{b}=27 \mathrm{kV}$. It is clear that two different frequencies exist. The middle and the lower traces show interferometer output patterns, when the gate pulse is adjusted to sample the output when $V_{b}=19.5$ and $V_{b}$ $=27 \mathrm{kV}$, respectively. The measured frequencies are 395 and $392 \mathrm{GHz}$. It is clear from these measurements that these frequencies are not present simultaneously. The switching of the cathode voltage $V_{b}$ results in the switching of the outpus frequency $f$.

The measured frequencies (395 and $392 \mathrm{GHz}$ ) are

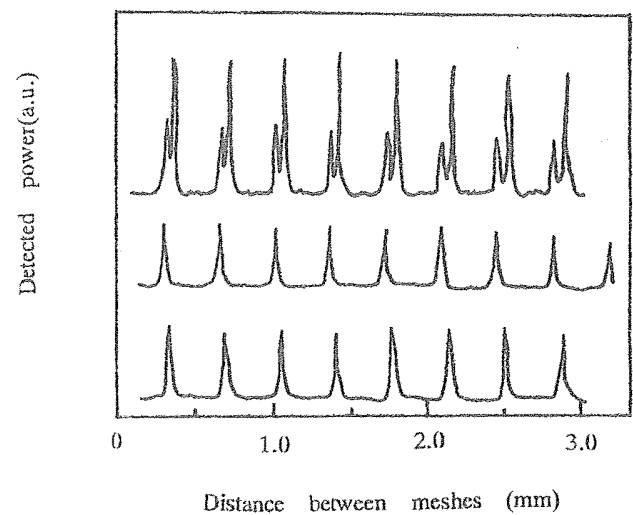

FIG. 5. Fabry-Perot interferometer patterns. $B_{0}=14.6 \mathrm{~T}, V_{b 1}=19.5 \mathrm{kV}$, and $V_{b 2}=27.0 \mathrm{kV}$. Upper trace: Ontput power, when the gate pulse covers both phases of $V_{b}=V_{b 1}$ and $V_{b}=V_{b 2}$. Middle trace: Output power at $V_{b}=V_{b 1}$ is sarmpled. Lower trace: Output power at $V_{b}=V_{b 2}$ is sampled.

slightly higher than the calculated values ( 391 and $387 \mathrm{GHz}$ ) shown in Table I. These differences may come from the measurement accuracy of the Fabry-Perot interferometer.

\section{EXPERMENTAL AND SIMULATION RESULTS FOR THE FPREQUENCY STEP-SWTCHWNG BETWEEN A FUNDARENTAL MODE AND A SEGOND HARMONIC} MODE

Next, we tried to switch the operating mode between a fundamental and a second hamonic. In this case, the frequency change is much more dramatic than in the previous case. A frequency step of several hundred $\mathrm{GH}_{z}$ has been achieved. We choose the $T F_{421}$ mode as the fundamental mode and the $T E_{161}$ mode as the second harmonic mode. The magnetic field intensity was chosen so that the beam voltages required for both modes were very close.

Figure 6 shows the computer simulation results of beam currents $I_{b}$ for Gyrotron FU XII, which are necessary to sustain several output powers for both modes, as functions of the cathode voltage $V_{b}$. The field intensity is set at the constant value of $8.12 \mathrm{~T}$. These results suggest that the switching between the two modes is possible by switching the cathode voltage $V_{b}$.

Figure 7 shows the distributions of high-frequency electric fields in the cavity along the radial direction. The injection radius of beam electrons $R_{\text {inj }}$ is adjusted at $0.75 \mathrm{~mm}$, by adjusting the intensity of magnetic field in the gun region. This injection point of the beam is suitable for both excitations of the fundamental and the second harmonic modes.

A typical experimental result is shown in Fig. 8 . The upper trace shows a high-voltage pulse applied to a gun cathode. It is modulated by a square wave whose frequency is 5 $\mathrm{kHz}$. This results in the switching of the cathode voltage $V_{b}$ between $V_{b 1}=24.2$ and $V_{b 2}=28.8 \mathrm{kV}$. The lower trace shows the output power measured by a pyroelectric detector.

Figure 9 shows Fabry-Perot interferometer patterns for frequency measurements. Figure 9(a) shows the interferometer pattern where the output power at $V_{b}=V_{b 1}$ is sampled 


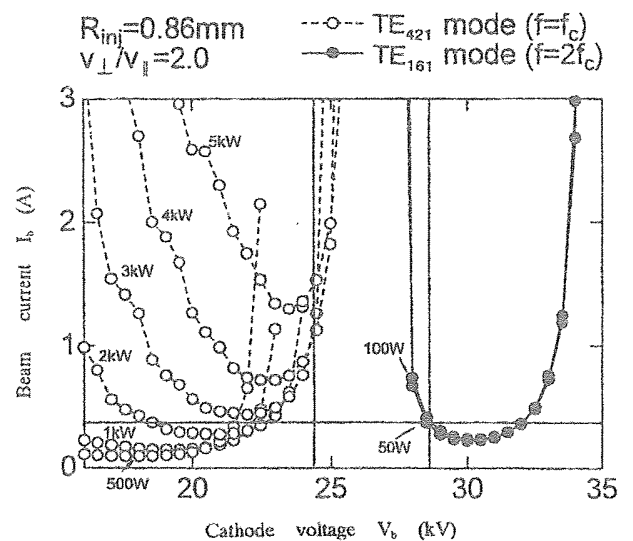

FIG. 6. Simulation results for beam currents $I_{b}$ to sustain constant output powers of several levels as functions of the cathode voltage $V_{b}$. Two cavity modes, $T E_{421}$ mode at the fundamental operation and $T E_{161}$ mode at the second harmonic operation, are studied. The magretic field intensity $b_{0}$ is kept at the consfant value of $8.12 \mathrm{~T}$.

and Fig. 9(b) where the output power at $V_{b}=V_{b 2}$ is sampled. It is clear that the fundamental mode is excited when $V_{b}$ $=V_{b 1}$ and the second hamonic mode when $V_{b}=V_{b 2}$. The fundamental corresponds to the $T E_{421}$ cavity mode at 223 $\mathrm{GHz}$ and the second harmonic to the $T E_{161}$ at $444 \mathrm{GHz}$. In this case, the frequency step is greater than $200 \mathrm{GHz}$. This is to be compared with the previous case of switching between two fundamental modes, where the frequency step was only a few $\mathrm{GH}$.

In both cases described in this section and the previous section, if the cathode voltage $V_{b}$ would be changed gxadually, the first cavity mode disappears once, and after then, the second mode appears, corresponding to the change of the operation conditions. In the present experiment, the process occurs very rapidly and, as a result, the rapid frequency stepswitching is realized.

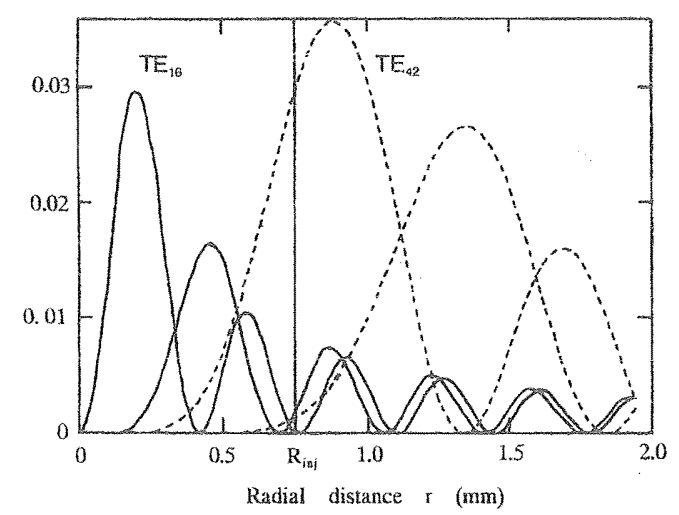

FIG. 7. The distributions of high-frequency electric field in the cavity as functions of a radial distance $r$. The injection radius $R_{\text {inf }}$ of beam electrons is adjusted at $0.75 \mathrm{~mm}$. The injection point is suitable for both excitations of $T E_{161}$ and $T E_{421}$ modes.

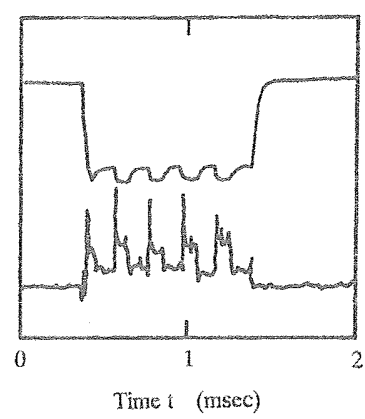

FIG. 8. Upper trace: High-voltage pulse applied to the electron gun cathode, modulated by a $5 \mathrm{kHz}$ square wave. Lower trace: Output power measured by a pyroelectric detector, $B_{0}=8.12 \mathrm{~T}, V_{b 1}=24.2 \mathrm{kV}$, and $V_{b 2}=28.8 \mathrm{kV}$.

A kind of hysteresis is pointed out in frequency step-switching. ${ }^{13}$ it is an interesting effect but our experiment up to the present has not proved such an effect. The more detailed experiment that we intend to do may make the process clear.

\section{SUNMTHAV}

The switching of the cathode voltage of electron gun $V_{b}$ results in the swiching of electron cyclotron frequency $f_{c}$. Gyrotrons operate near the electron cyclotron frequency $f_{c}$ or a harmonic $n f_{c}$. Therefore, if two cavity modes are ca" pable of being excited at the same magnetic field intensity, it is easy to switch from one cavity mode to the other by switching $V_{b}$. In this way, the frequency step-switching can be obtained. Following this principle, frequency stepswitching is analyzed by computer simulations and demonstrated experimentally for two cases using submillimeter wave gyrotrons in Fukui University, Gyrotrons FU III and FU IV.

In the first case, the switching is between two fundamental modes. The frequency step is only 3 or $4 \mathrm{GHz}$. In the second case, the switching is between a fundamental mode and a second harmonic mode. In this case, the frequency step is very much larger, more than $200 \mathrm{CHz}$

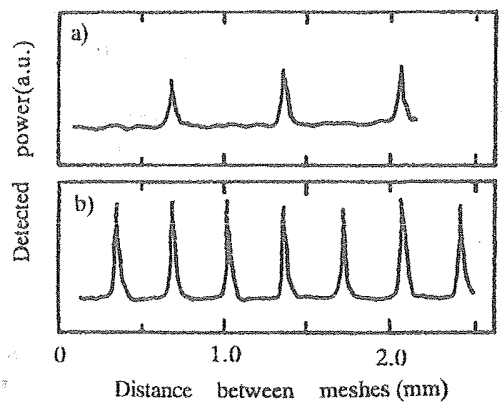

FIG. 9. Fabry-Perot interferometer patterns. $B_{0}=8.12 \mathrm{~T}, V_{b 1}=24.2 \mathrm{kV}$, and $V_{b 2}=28.8 \mathrm{kV}$. (a) The output power at $V_{b}=V_{b 1}$ is sampled. (b) The output power at $V_{b}=V_{b 2}$ is sampled. 
Frequency step-switching of gyrotron output can be utilized in a wide range of applications such as plasma diagnos tics, plasma beating, multichannel measurement of materials, and so on.

\section{ACKNOWLEDGMENT}

The work is supported partially by a Grant in Aid from the Ministry of Education, Science and Culture in Japan (Monbusho).

B. Müller, V. Erckmann, M. Thumm, R. Wilheim, D. Dorst, and W. Melkus, Proceedings of 13th Symposium on Fusion Technology, Varese 1984 (Pergamon, Oxford, 1984), pp. 811-816

${ }^{2}$ E. Borie, G. Dammertz, O. Dumbrajs, T. Geist, M. Kuntze, A. Möbius, H

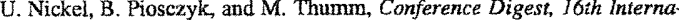
tional Conference on Infrared and Millimeter Waves, Lausanne, 26-30 August, edited by M. R. Siegrist, M. Q. Tran, and T. M. Tran (Centre de Researches en Physique des Plasmas, Lausanne, 1991), pp. 260-263. ${ }^{3}$ T. Idehara, Y. Shimizu, S, Makino, K. Ichikawa, T. Tatsukawa, I. Ogawa, and G. F. Brand, Phys. Plasmas 1, 461 (1994).

${ }^{4}$ T. Idehara, Y. Shimizu, S. Makino, K. Ichikawa, T. Tatsukawa, I. Ogawa, and G. F. Brand, int. J. Infrared Millim. Waves 18, 391 (1997).
${ }^{5}$ T. Idehara, K. Ichikawa, I. Ogawa, T. Tatsukawa, and G. F. Brand, Int. J. infrared Millim. Waves 19, 1607 (1998)

${ }^{6} \mathrm{~T}$. Idehara, M. Pereyaslavets, N. Nishida, K. Yoshida, and L. Ogawa, Phys. Rev. Lett. 81, 1973 (1998)

M. Pereyaslavets, T. Idehara, N. Nishida, K. Yoshida, and I. Ogawa, "Simulation and measurement of frequency modulation in submillimeter wave gyrotron," IEEE Trans. Plasma Sci. (to be published)

${ }^{8}$ O. Dumbrajs and G. S. Nusinovich, IEEE Trans. Plasma Sci. 20, 452 (1992).

${ }^{9}$ G. S. Nusinovich, B. Levish, T. M. Antonsen, Ir., and A. Bromborsby, Abstracts of Second Intemational Workshop on Strong Microwaves in Plasmas, Nizhniy Novgorod, Russia, 1993 (Russian Academy of Sciences, Nizhniy Novgorod, 1993), No. S-7.

${ }^{10} \mathrm{~T}$. Idehara, Y. Shimizu, S. Makino, K. Ichikawa, T. Tatsukawa, I. Ogawa, and G. F. Brand, Phys. Plasraas 1, 1774 (1994)

${ }^{~ " Y . ~ S h i m i z u, ~ S . ~ M a k i n o, ~ K . ~ I c h i k a w a, ~ T . ~ K a n e m a k i, ~ I . ~ O g a w a, ~ T . ~ T a t " ~}$ sukawa, and T. Idehara, Phys. Placmas 2, 2110 (1995).

${ }^{12}$ T. Idehara, Y. Shimizu, K. Ichikawa, S. Makino, K. Shibutani, K. Kurahashi, T. Tatsukawa, I. Ogawa, Y. Okazaki, and T. Okamoto, Phys. Plasmas 2, 3246 (1995).

${ }^{13}$ O. Dumbrajs, V. I. Khizhnyak, A. B. Pavelyev, and J. Heikkinen, Conference Digest, 20th International Conference on Infrared and Millimeter Waves, Orlando, Florida, 11-14 December, edited by R. J. Temkin (Institute of Electrical and Electronics Engineers Oriando Section, 1995), pp. $546-547$. 